\title{
Pathogenèse de l'endométriose ${ }^{1}$ \\ Pathogenesis of endometriosis
}

\author{
M. Nisolle ${ }^{\mathrm{a}, \mathrm{b}}$, M.-L. Alvarez ${ }^{\mathrm{b}}$, M. Colombo ${ }^{\mathrm{b}}$, J.-M. Foidart ${ }^{\mathrm{a}, \mathrm{b}}$ \\ ${ }^{a}$ Département de gynécologie-obstétrique, université de Liège, hôpital de la Citadelle, 1, boulevard du $12^{e}$ de Ligne, 4000 Liège, Belgique \\ ${ }^{b}$ Laboratoire de biologie des tumeurs et du développement, université de Liège, CHU Sart-Tilman, B23, 4000 Liège, Belgique
}

\section{Résumé}

L'endométriose, définie par la présence ectopique de tissu endométrial, est une affection bénigne responsable d'infertilité ou de douleurs pelviennes. Un diagnostic précoce fondé sur une anamnèse minutieuse et un examen clinique bien conduit permet une prise en charge précoce. Le traitement médical n'est pas approprié dans tous les cas, faisant place à la cœlioscopie opératoire dont la morbidité est en relation avec la sévérité de la lésion.

L'implantation ectopique de cellules endométriales est possible grâce à des interactions complexes entre le tissu hôte et les cellules épithéliales endométriales. Les conditions nécessaires au développement de l'endométriose sont une prolifération estrogénodépendante des cellules endométriales, une induction d'angiogenèse et d'une lymphangiogenèse. Les principaux acteurs cellulaires et moléculaires de l'angiogenèse, de la lymphangiogenèse et de la fibrose doivent être identifiés afin de permettre le développement de nouvelles stratégies thérapeutiques de l'endométriose.

Mots clés : Endométriose ; Angiogenèse ; Lymphangiogenèse ; Inflammation ; Fibrose

\begin{abstract}
Endometriosis, defined by the development of endometrial tisue outside the uterus, is a benign disease responsible for infertility and pelvic pain. The diagnosis based on a detailed gynecological history and a careful clinical examination should be done as early as possible in order to treat patients correctly. Medical treatment is not appropriate in all cases and surgical treatment should be proposed but morbidity is related to the severity of the lesion. Ectopic implantation of endometrial cells needs complex interactions between host tissue and epithelial endometrial cells. The conditions for the development of endometriosis are estrogeno-dependent growth of endometrial cells, induction of angiogenesis and lymphangiogenesis. Principal cellular and molecular factors of angiogenesis, lymphangiogenesis and fibrosis should be identified in order to develop new therapeutic strategies of endometriosis.
\end{abstract}

Keywords: Endometriosis; Angiogenesis; Lymphangiogenesis; Inflammation; Fibrosis

\section{Introduction}

L'endométriose se définit par la présence de tissu endométrial composé de glandes et de stroma cytogène en dehors de la cavité utérine. Cette affection bénigne atteint 10 à $15 \%$ des femmes en âge de reproduction et peut être responsable soit d'infertilité, soit de douleurs pelviennes chroniques parfois sévères et invalidantes, avec une répercussion possible sur la qualité de vie des femmes.

Les localisations les plus fréquentes concernent la sphère génitale et notamment le péritoine, les ovaires et le culde-sac de Douglas. La théorie du reflux menstruel et de la transplantation, proposée par Sampson en 1927, permet d'expliquer le développement de l'endométriose au niveau du pelvis [1]. Cependant, elle ne permet pas d'expliquer d'autres localisations plus rares, comme les poumons ou le cerveau, sans communication avec la

\footnotetext{
${ }^{1}$ Conférence d'actualité.
} 
cavité pelvienne [2]. La dissémination par voie vasculaire ou lymphatique est une théorie permettant de comprendre le développement de ces lésions à distance dont la fréquence est estimée à $5 \%$ des lésions d'endométriose. Des travaux récemment publiés suggèrent le rôle des cellules souches dans la progression des lésions d'endométriose induites chez la souris [3].

L'implantation ectopique de cellules endométriales est possible grâce à des interactions complexes entre le tissu hôte et les cellules épithéliales endométriales. Les conditions nécessaires au développement de l'endométriose sont la survie des cellules endométriales en dehors de la cavité utérine, leur adhésion, leur infiltration, une prolifération estrogénodépendante, l'induction d'angiogenèse et de lymphangiogenèse. Le tissu hôte est, par ailleurs, responsable du développement d'une fibrose secondaire à l'implantation de ces cellules endométriales épithéliales et stromales.

\section{Formes cliniques de l'endométriose}

L'endométriose péritonéale superficielle peut présenter plusieurs aspects macroscopiques lors de la cœlioscopie diagnostique. La lésion superficielle typique est de couleur noire, en forme de rétraction ou de petit nodule kystique. Les lésions atypiques sont des lésions rouges, des vésicules claires, des plaques blanches, des poches péritonéales ou des zones jaunâtres.

En 1993, une classification de l'endométriose péritonéale a été proposée en lésions rouges, noires et blanches, fondée sur leurs différences d'aspect macroscopique et de vascularisation stromale [4]. Cette classification permet de différencier les lésions récemment développées, richement vascularisées et inflammatoires, des lésions plus anciennes responsables d'une réaction fibrotique. Cette hypothèse d'évolution des lésions rouges en lésions noires et ensuite en lésions blanches a été ultérieurement confirmée par la mise en évidence d'une angiogenèse différente au sein de ces différents types de lésions péritonéales $[5,6]$.

L'endométriose ovarienne se présente le plus souvent sous forme de kyste, appelé endométriome. Il contient un liquide épais de couleur chocolat, et il est fréquemment adhérent au péritoine de la fossette ovarienne et éventuellement à d'autres organes adjacents (utérus, ligaments utérosacrés, rectum, intestin grêle, appendice).

L'endométriose sous-péritonéale profonde inclut toutes les lésions rétropéritonéales ou infiltrant les viscères abdominaux ou pelviens (rectum, uretère, intestin grêle, etc.). Les lésions profondes sont essentiellement localisées au niveau des ligaments utérosacrés, la paroi vaginale postérieure, la paroi vésicale et la face antérieure du rectum ou de la jonction rectosigmoïdienne.

\section{Histologie de l'endométriose}

Le critère diagnostique de l'endométriose repose sur l'observation de tissu endométrial comportant les deux composantes élémentaires (glandes et stroma). Le saignement au sein des foyers d'endométriose, concomitant de la menstruation, se traduit par des suffusions hémorragiques dans le chorion cytogène et dans la lumière glandulaire, et s'accompagne d'une réaction inflammatoire à prédominance macrophagique. La réaction inflammatoire aboutit à une fibrose réactionnelle périlésionnelle variable.

D'un point de vue histologique, les lésions profondes nodulaires sont caractérisées par la présence de glandes endométriales, de cellules endométriales stromales raréfiées et d'une hyperplasie fibromusculaire importante. Ces lésions sont également le siège d'une angiogenèse et d'une lymphangiogenèse comme dans les processus tumoraux $[7,8]$.

\section{Analogie avec le processus tumoral}

De même que pour le développement du processus tumoral, l'implantation ectopique de cellules endométriales nécessite des interactions complexes entre le tissu hôte et les cellules épithéliales endométriales et probablement les cellules stromales. Il a été clairement démontré que la capacité d'une cellule tumorale de métastaser dépend de ses interactions avec les cellules hôtes qui sont responsables de la croissance et de la survie cellulaire tumorale, d'angiogenèse et d'invasion [9]. Dans le cadre de l'endométriose, les cellules endométriales doivent également survivre en dehors de la cavité utérine, s'implanter, échapper au système immunitaire, induire une angiogenèse, une lymphangiogenèse, et ensuite proliférer [10]. Au cours de ces processus, les cellules endométriales interagissent avec les cellules hôtes des tissus avoisinants. Celles-ci incluent les cellules épithéliales du mésothélium et de l'ovaire, les fibroblastes, les cellules endothéliales, les leucocytes et les macrophages. 


\section{Adhésion des cellules endométriales}

Des modèles in vivo et in vitro ont permis de démontrer la capacité des cellules endométriales d'adhérer au péritoine. L'induction des lésions d'endométriose peut être obtenue avec succès chez le babouin et la souris nue. Ce modèle murin représente le modèle le plus largement étayé dans la littérature, le taux de succès de cette transplantation d'endomètre humain chez la souris pouvant atteindre jusqu'à $87 \%$ (33 à 87\%) [11-13].

La transplantation d'endomètre humain dans la cavité péritonéale ou en sous-cutané permet d'obtenir des lésions d'endométriose très rapidement, l'interaction entre les cellules stromales et glandulaires étant une condition indispensable au succès de cette transplantation [14]. Un rôle distinct a été attribué aux cellules glandulaires et stromales : les cellules glandulaires révélant une activité proliférative intense seraient responsables de la croissance des lésions, tandis que les cellules stromales seraient responsables de la première étape d'adhésion des cellules endométriales [15].

L'interaction entre les cellules épithéliales et stromales dans le développement de l'endométriose a également été démontrée par l'absence de formation de lésions lorsque les cellules endométriales, épithéliales et stromales sont injectées séparément dans la cavité péritonéale de souris. En revanche, une injection concomitante de ces deux types cellulaires permet d'obtenir des lésions d'endométriose dans $50 \%$ des cas [14]. Cette interaction entre les cellules épithéliales et stromales implique probablement un dialogue entre ces deux types cellulaires par l'intermédiaire de facteurs de croissance, de cytokines et/ou de chimiokines.

Par ailleurs, la contribution des cellules stromales à l'adhésion des cellules épithéliales est sans doute en relation avec un remodelage de la matrice extracellulaire sous l'action de protéases et avec la formation d'un micro environnement approprié à l'implantation des cellules épithéliales, ainsi qu'à leur réorganisation en glandes endométriales. Ce type d'interaction, épithélium-stroma, a déjà été démontré pour l'implantation des cellules tumorales [16].

De nombreux travaux in vitro ont permis également de démontrer la capacité des cellules endométriales d'adhérer à des fragments de membranes chorio-allantoïdiennes de poulet ou de péritoine de souris [17,18]. Des travaux de Witz et al. ont permis d'observer que l'adhésion entre les cellules endométriales et le mésothélium était possible en une heure, et qu'en 24 heures, ces cellules endométriales étaient capables d'envahir totalement le mésothélium [19].

Le rôle du mésothélium, en tant que barrière s'opposant à l'adhésion des cellules endométriales, proposé par certaines équipes, n'a pas été confirmé par d'autres travaux mettant en évidence une adhésion rapide et facile des cellules endométriales sur un mésothélium intact. Un défect épithélial, secondaire à un traumatisme tissulaire, ne semble donc pas être une condition indispensable à l'adhésion des cellules endométriales et à la formation de lésions d'endométriose.

Des travaux in vitro ont déterminé les cytokines et les facteurs de croissance favorisant cette étape d'adhésion : l'interleukine 1 (IL-1), l'interleukine 6 (IL-6), le TNF- $\alpha$ et le TGF- $\beta$ sont responsables d'une augmentation de l'adhésion des cellules endométriales au niveau de péritoine murin [18].

Les acteurs moléculaires de cette adhésion des cellules endométriales au niveau du péritoine sont la laminine et la fibronectine, deux glycoprotéines d'adhésion qui exercent leur action par l'intermédiaire de récepteurs de la famille des intégrines. L'expression des récepteurs de la fibronectine ( $\alpha 4 \beta 1$ et $\alpha 5 \beta 1)$ persiste au niveau des glandes endométriosiques, alors qu'elle n'est pas observée au niveau des glandes endométriales eutopiques en fin de phase sécrétoire [20]. Ces récepteurs de la fibronectine jouent donc très probablement un rôle dans la persistance des lésions d'endométriose.

\section{Infiltration des cellules endométriales}

Afin de pénétrer profondément dans le tissu hôte, les cellules épithéliales endométriales doivent dans un premier temps se détacher des cellules voisines avec lesquelles elles sont en connexion par les cadhérines-E. Les cadhérines sont des molécules responsables de l'adhésion intercellulaire et sont exprimées au niveau des cellules épithéliales endométriales. Des différences de capacité d'invasion entre les cellules endométriales de patientes endométriosiques ou sans endométriose ont été mises en évidence sur un modèle in vitro de collagène [21]. Une proportion plus élevée de cellules épithéliales négatives pour la cadhérine-E et potentiellement invasives a été notée au niveau des cellules épithéliales endométriosiques comparativement aux cellules épithéliales endométriales. 
On observe, au niveau des cancers épithéliaux, une perte d'expression de la cadhérine-E responsable de l'acquisition d'un phénotype de type invasif Par analogie, ce même mécanisme pourrait être proposé pour le développement de l'endométriose, si des travaux confirmaient ces mêmes observations.

\section{Prolifération des cellules endométriales}

Les estrogènes, responsables de la croissance et de la différenciation de l'endomètre eutopique, exercent le même rôle au niveau de l'endomètre ectopique. La mise en évidence essentiellement des récepteurs aux estrogènes de type $\alpha$, au niveau des lésions d'endométriose, permet d'évoquer leur rôle essentiel dans le développement et la croissance de l'endométriose.

Une production locale d'estradiol, au sein des implants d'endométriose, est également responsable de la croissance de la lésion, indépendamment du taux plasmatique d'estrogènes. Cette production locale d'estrogènes est due à la conversion des androgènes en estradiol sous l'effet de l'enzyme aromatase. Au niveau de l'endomètre eutopique, l'aromatase n'est pas exprimée contrairement à ce que l'on observe au niveau des cellules stromales endométriosiques [22]. Cette observation permet d'expliquer une production locale d'estradiol et une croissance des lésions, en dépit de traitements supprimant la production d'estrogènes d'origine ovarienne.

L'aromatase n'est pas seulement produite par les cellules de la granulosa, mais aussi par la peau, le cerveau, les adipocytes et par l'endomètre ectopique.

L'administration d'inhibiteurs d'aromatase, sur un modèle murin d'endométriose, a permis de démontrer une diminution significative du volume des implants endométriosiques par rapport aux souris non traitées [23].

Nos travaux de transplantation d'endomètre chez la souris ont permis également de démontrer l'expression de l'aromatase au niveau des cellules glandulaires endométriosiques. La présence de l'aromatase est observée dans toutes les lésions d'endométriose expérimentale dès le troisième jour après la greffe, alors que son absence est confirmée au niveau de l'endomètre eutopique [résultats non publiés].

L'administration d'inhibiteurs d'aromatase chez la souris a également induit une diminution de l'activité proliférative cellulaire confirmant le rôle de cette enzyme dans la prolifération de l'endométriose. Ces travaux sont actuellement en cours et des études cliniques devraient confirmer l'efficacité de cette nouvelle stratégie thérapeutique dans le traitement de l'endométriose.

Chez la femme, l'administration d'anti-aromatase entraine une augmentation du recrutement folliculaire chez les normo-ovulantes et une augmentation du taux sanguin d'estradiol.

Aucune étude n'a permis de démontrer qu'il existait un blocage ovarien suffisant après l'administration d'inhibiteurs de l'aromatase. Leur administration à des femmes non ménopausées devrait donc être associée à d'autres traitements freinateurs de l'axe hypothalamo-hypophyso-ovarien.

\section{Angiogenèse}

La formation de nouveaux vaisseaux est une étape essentielle au développement et à la survie de l'endométriose. Les principaux acteurs cellulaires de l'angiogenèse sont les cellules endothéliales qui lorsqu'elles sont activées, prolifèrent, dégradent leur membrane basale, migrent dans la matrice extracellulaire environnante et s'organisent en bourgeons vasculaires.

Le liquide péritonéal des patientes présentant de l'endométriose contient des substances solubles impliquées dans l'angiogenèse comme notamment le VEGF [24]. Le VEGFA est, en effet, une cytokine angiogénique très puissante, produite en grande quantité par les cellules épithéliales et stromales des lésions d'endométriose. Son niveau d'expression est directement corrélé au degré de développement des lésions.

De plus, le VEGF induit par une probable hypoxie et/ou l'inflammation, est également responsable d'une anomalie fonctionnelle de ces vaisseaux, car il provoque une augmentation de la perméabilité vasculaire et favorise l'extravasation de fibrine et une attraction secondaire des macrophages.

Divers facteurs angiogéniques sont, en outre, produits par les macrophages péritonéaux, les cellules endométriales menstruelles régurgitées et/ou les lésions d'endométriose elles-mêmes. Des études immunohistochimiques ont permis de démontrer un marquage intense du VEGF au niveau de l'épithélium et du 
stroma des implants d'endométriose, essentiellement au niveau des lésions d'aspect rouge.

Au niveau des lésions profondes, la majorité des vaisseaux localisés dans le stroma sont dépourvus de péricytes, immatures et fragiles. Ils sont essentiellement localisés à proximité des glandes endométriales comme l'a démontré une analyse morphométrique par analyse d'images assistée par ordinateur [8,25]. Cette localisation préférentielle des vaisseaux suggère un rôle paracrine des cellules épithéliales.

Si les vaisseaux sont immatures, ils sont également une cible idéale pour une thérapie anti-angiogène comme l'ont démontré des travaux réalisés sur un modèle murin [26]. En effet, une diminution significative du nombre de lésions d'endométriose a été notée après la transplantation d'endomètre chez la souris, sous l'effet d'agents angiostatiques. Ces agents angiostatiques diminuent également de manière significative la densité des microvaisseaux et le nombre de vaisseaux immatures.

Il a également été récemment démontré, à partir d'un modèle murin, que la revascularisation des implants des fragments d'endomètre transplantés survient après cinq à huit jours [27]. Le développement d'une angiogenèse d'origine murine est une condition indispensable à la survie de la lésion d'endométriose. L'origine de ces cellules endothéliales murines pourrait soit provenir de vaisseaux proches de la lésion, soit de cellules endothéliales progénitrices d'origine médullaire, attirées par l'implant. Des travaux récents permettent de suggérer que les cellules souches ont un rôle dans la progression des lésions d'endométriose induites chez la souris [3].

Les métalloprotéinases matricielles (MMPs) représentent une famille d'endopeptidases favorisant la dégradation et le turnover des protéines de la matrice extracellulaire [28]. Dans l'endomètre, les MMPs sont responsables du remodelage de la matrice lors de la phase périmenstruelle. L'expression cyclique des MMPs par l'endomètre humain pourrait jouer un rôle dans le processus d'invasion indispensable au développement de l'endométriose. Les MMPs interviennent également au niveau de l'angiogenèse, car elles permettent la protéolyse de la membrane basale sous-endothéliale et la migration des cellules endothéliales à travers la matrice extracellulaire. La détection des MMP-2 et MMP-9, au niveau des lésions d'endométriose, confirme cette hypothèse [29]. Des données impliquent une corrélation entre la surexpression de MMPs et la sous-expression de leurs inhibiteurs (TIMPs) d'une part, et l'importance de la vascularisation de la lésion d'autre part [30].

\section{Lymphangiogenèse}

La formation de nouveaux vaisseaux lymphatiques au sein des lésions d'endométriose profonde a été récemment démontrée par un immunomarquage au D2-40 [8]. Il s'agit bien d'une lymphangiogenèse, car les vaisseaux lymphatiques sont absents de la couche fonctionnelle, et ne sont présents que dans la couche basale de l'endomètre [31]. Une localisation préférentielle de ces vaisseaux lymphatiques a également été observée à proximité des glandes endométriales, dont le rôle paracrine doit encore être étudié.

Il n'est pas exceptionnel de détecter des ganglions lymphatiques infiltrés par des cellules endométriales lors de l'analyse de pièces opératoires de résection digestive réalisée pour une endométriose colorectale infiltrante [32]. La dissémination de l'endométriose digestive à distance de la lésion primitive pourrait également être due à une dissémination par voie lymphatique, et expliquer de cette manière la multifocalité des lésions fréquemment décrites.

Une corrélation entre l'angiogenèse et la lymphangiogenèse au niveau des lésions profondes d'endométriose suggère que non seulement l'angiogenèse est primordiale pour le développement de l'endométriose, mais également que la lymphangiogenèse est impliquée dans l'évolution de l'endométriose.

\section{Fibrose et inflammation : principaux acteurs cellulaires}

L'inflammation est un stimulus nécessaire pour initier le phénomène de cicatrisation au cours de laquelle des cytokines sécrétées par des leucocytes stimulent la prolifération et l'activation des myofibroblastes. Les myofibroblastes activés peuvent également secréter des cytokines pro-inflammatoires, recruter des cellules inflammatoires et amplifier ainsi la réponse fibrotique en un cercle vicieux. De plus, certains myofibroblastes issus de cellules progénitrices de la moelle osseuse semblent se différencier directement à partir de monocytes circulants. Les macrophages jouent un rôle central non seulement dans la réponse fibrotique, mais également dans la résolution de la fibrose.

La connaissance des principaux acteurs cellulaires et moléculaires de la relation entre l'inflammation et la fibrose est primordiale dans le cadre du développement de nouvelles thérapeutiques spécifiques et efficaces de 
l'endométriose.

En effet, l'endométriose est une affection inflammatoire dont l'évolution naturelle se fait vers le développement d'une fibrose, qui au niveau péritonéal est le plus souvent synonyme d'une guérison spontanée des lésions isolées ou de la formation de brides adhérentielles entre zones lésées. À d'autres endroits, comme au niveau de la paroi rectale, elle est responsable du développement d'un noyau fibreux douloureux.

Les médiateurs cellulaires de la fibrose sont les myofibroblastes et les macrophages.

Les myofibroblastes activés sont responsables des trois étapes essentielles de la fibrose active à savoir, la production de la matrice, son remodelage et sa contraction. Lors de la progression de la fibrose, on observe une augmentation de l'expression des MMPs (MMP-2, MMP-3 et MMP-9), ainsi que des TIMP (TIMP-1 et TIMP2). Les myofibroblastes activés expriment tous les composants nécessaires à la dégradation matricielle.

Les macrophages jouent un rôle prédominant dans le développement de la fibrose puisqu'ils sont responsables de la régulation des processus de fibrinogenèse et de fibrinolyse. Les macrophages sont également responsables de l'activation des fibrocytes en myofibroblastes.

Au niveau des lésions d'endométriose profonde, le développement excessif de fibrose, ainsi que la raréfaction des cellules stromales d'origine endométriale restent à l'heure actuelle des phénomènes non élucidés.

La transplantation d'endomètre humain chez des souris immunodéficientes, soit de type nue (nude mouse), soit de type SCID, nous a permis de suggérer un rôle possible des cellules immunitaires dans le développement de cette fibrose péri-endométriale. En effet, consécutivement à la transplantation d'endomètre humain chez les souris totalement immunodéficientes (souris SCID), on observe une fibrose réactionnelle limitée (Fig. 1). En revanche, de manière surprenante chez les souris partiellement immunodéficientes (souris nues), une fibrose périglandulaire majeure et une raréfaction des cellules stromales humaines sont observées une semaine après la transplantation d'endomètre. Ces lésions d'endométriose sont caractérisées par une disparition des cellules stromales d'origine humaine, progressivement remplacées par du tissu fibromusculaire (Fig. 2). La différence essentielle entre ces deux types de souris consiste en la présence de lymphocytes B chez les souris nues. Le rôle exact des lymphocytes B dans le développement de la fibrose doit encore être élucidé. Une relation entre les macrophages et le développement de la fibrose dans l'endométriose est également évoquée, mais doit encore être confirmée.

Le rôle du tissu hôte dans le développement de cette fibrose a été mis en évidence dans ce modèle animal grâce à la technique d'hybridation in situ en fluorescence (FISH) détectant le centromère du chromosome X d'origine humaine. Nous avons démontré l'absence de cellules positives pour le FISH au niveau de la réaction fibrotique, alors que les cellules glandulaires et stromales d'origine humaine sont positives. Ces résultats confirment ainsi l'origine murine de la fibrose dans notre modèle animal et suggèrent le rôle du tissu hôte secondaire à l'implantation de cellules endométriales.

Fig. 1. Développement d'une lésion d'endométriose suite à la transplantation d'endomètre humain chez la souris SCID. La fibrose réactionnelle est limitée.

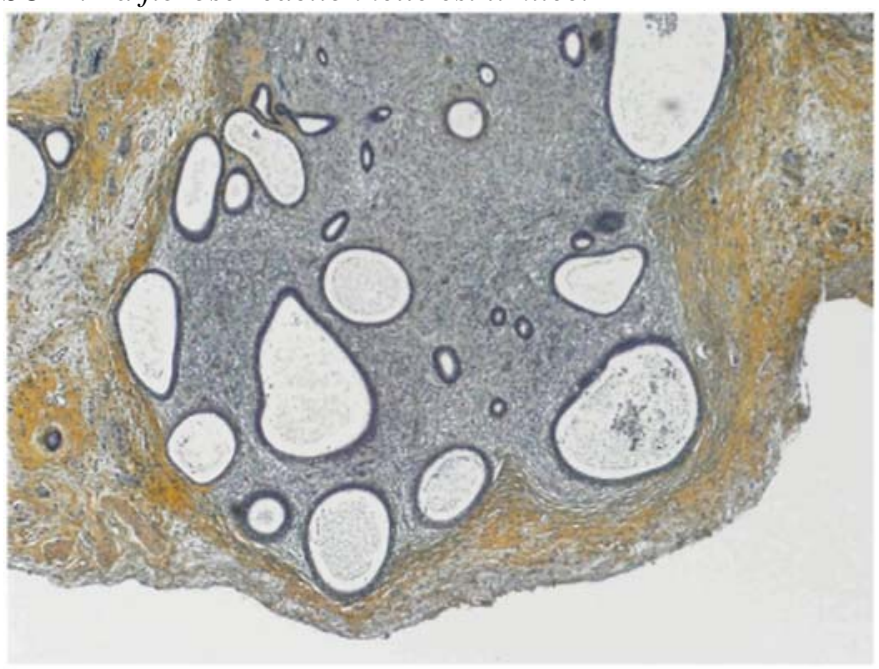


Fig. 2. Développement d'une lésion d'endométriose suite à la transplantation d'endomètre humain chez la souris nue. La fibrose périglandulaire d'origine murine est importante, les cellules stromales humaines se raréfient.

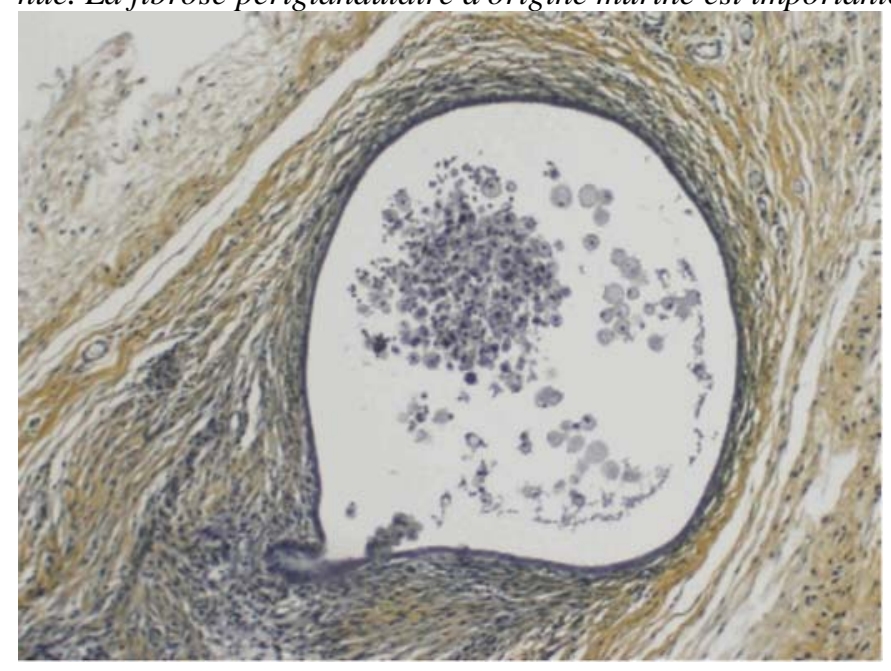

\section{Conclusion}

Le développement de l'endométriose présente une analogie avec le processus tumoral au cours duquel les cellules endométriales s'implantent, échappent au système de défense du tissu hôte et prolifèrent grâce à une production locale d'estradiol par une aromatase. Les lésions progressent grâce à une angiogenèse et peuvent migrer à distance grâce au développement d'une lymphangiogenèse. L'angiogenèse et la lymphangiogenèse sont probablement liées à une stimulation paracrine à partir des cellules glandulaires, alors que la fibrose réactionnelle pourrait être expliquée par l'inflammation et la participation des macrophages.

Il est primordial de déterminer les acteurs moléculaires responsables de l'établissement des lésions d'endométriose, de leur prolifération et de l'extension à distance. Ces connaissances permettront de développer des stratégies thérapeutiques visant de nouvelles cibles moléculaires et cellulaires dans le but d'inhiber la réaction cellules endométriales-tissu hôte.

\section{Références}

[1] Sampson JA. Peritoneal endometriosis due to the menstrual dissemination of endometrial tissue into the peritoneal cavity. Am J Obstet Gynecol 1927;14:422-69.

[2] Nisolle M, Pasleau F, Foidart JM. L'endométriose extragénitale. Recommandations pour la pratique clinique. J Gynecol Obstet Biol Reprod (Paris) 2007;36:173-8.

[3] Du H, Taylor HS. Contribution of bone marrow derived stem cells to endometrium and endometriosis. Stem Cells 2007(April):26.

[4] Nisolle M, Casanas-Roux F, Anaf V, Mine JM, Donnez J. Morphometric study of the stromal vascularization in peritoneal endometriosis. Fertil Steril 1993;59:681-4

[5] Nisolle M. Peritoneal, ovarian and rectovaginal endometriosis are three distinct entities [thèse d'agrégation de l'enseignement Supérieur]. Louvain : Université catholique de Louvain; 1996.

[6] Nisolle M, Donnez J. Peritoneal endometriosis, ovarian endometriosis, and adenomyotic nodules of the rectovaginal septum are three different entities. Fertil Steril 1997;68:585-96.

[7] Nisolle M, Alvarez ML, Nervo P, Foidart JM. Endométriose — Les conceptions physiologiques actuelles. Reprod Hum et Horm 2006; 19: 343-51.

[8] Nervo P, Nisolle M, Blacher S, Brichant JF, Foidart JM. Angiogenesis and lymphangiogenesis in deep infiltrating endometriosis. 2007 (soumis à Fertility and Sterility).

[9] Fidler IJ. The pathogenesis of cancer metastasis: the "seed and soil" hypothesis revisited. Nat Rev Cancer 2003;3:453-8. 
Published in: Gynécologie Obstétrique \& Fertilité (2007), vol. 35, iss.9, pp. 898-903

Status: Postprint (Author's version)

[10] Nisolle M, Foidart JM. Pathogenesis of peritoneal endometriosis. In: Sutton C, Jones K, Adamson GD, editors. In modern management of endometriosis. London, New York: Taylor \& Francis Group; 2006. p. 25-40.

[11] Aoki D, Katsuki Y, Shimizu A, Kakinuma C, Nozawa S. Successful heterotransplantation of human endometrium in SCID mice. Obstet Gynecol 1994;83:220-8.

[12] Bergqvist A, Jeppsson S, Kullander S, Ljungberg O. Human endometrium transplanted into nude mice. Histologic effects of various steroid hormones. Am J Pathol 1985;119:336-44.

[13] Nisolle M, Casanas-Roux F, Marbaix E, Jadoul P, Donnez J. Transplantation of cultured expiants of human endometrium into nude mice. Hum Reprod 2000;15:572-7.

[14] Beliard A, Noel A, Goffin F, Frankenne F, Foidart JM. Role of endocrine status and cell type in adhesion of human endometrial cells to the peritoneum in nude mice. Fertil Steril 2002;78:973-8.

[15] Nisolle M, Casanas-Roux F, Donnez J. Early-stage endometriosis: adhesion and growth of human menstrual endometrium in nude mice. Fertil Steril 2000;74:306-12.

[16] Noel A, Kebers F, Maquoi E, Foidart JM. Cell-cell and cell-matrix interactions during breast cancer progression. Curr Top Pathol 1999;93:183-93.

[17] Groothuis PG, Koks CA, De Goeij AF, Dunselman GA, Arends JW, Evers JL. Adhesion of human endometrium to the epithelial lining and extracellular matrix of amnion in vitro: an electron microscopic study. Hum Reprod 1998;13:2275-81.

[18] Beliard A, Noel A, Goffm F, Frankenne F, Foidart JM. Adhesion of endometrial cells labelled with ${ }^{111}$ Indium-tropolonate to peritoneum: a novel in vitro model to study endometriosis. Fertil Steril 2003;79(Suppl 1):724-9.

[19] Witz C, Thomas MR, Montoya-Rodriguez IA, Nair AS, Centonze VE, Schenken RS. Short-term culture of peritoneum expiants confirms attachment of endometrium to intact peritoneal mesothelium. Fertil Steril 2001; 75:385-90.

[20] Beliard A, Donnez J, Nisolle M, Foidart JM. Localization of laminin, fibronectin, E-cadherin, and integrins in endometrium and endometriosis. Fertil Steril 1997;67:266-72.

[21] Gaetje R, Kotzian S, Herrmann G, Baumann R, Starzinski-Powitz A. Non malignant epithelial cells, potentially invasive in human endometriosis, lack the tumor suppressor molecule E-cadherin. Am J Pathol 1997; 150:461-7.

[22] Bulun SE, Zeitoun KM, Takayama K, Sasano H. Molecular basis for treating endometriosis with aromatase inhibitors. Hum Reprod Update 2000;6:413-8.

[23] Fang Z, Yang S, Gurates B, Tamura M, Simpson E, Evans D, et al. Genetic or enzymatic disruption of aromatase inhibits the growth of ectopic uterine tissue. J Clin Endocrinol Metab 2002;87:3460-6.

[24] Oosterlynck DJ, Meuleman C, Sobis H, Vandeputte M, Koninckx PR. Angiogenic activity of peritoneal fluid from women with endometriosis. Fertil Steril 1993;59:778-82.

[25] Ravet S, Labied S, Blacher S, Frankenne F, Munaut C, Fridman V, et al. Endometrial vessel maturation in women exposed to levonorgestrel-releasing intrauterine system for short or prolonged period of time. Hum Reprod 2007 (accepté pour publication).

[26] Nap AW, Griffioen AW, Dunselman GA, Bouma-Tersteege JC, Thijs-sen VL, Evers JL, et al. Antiangiogenesis therapy for endometriosis. J Clin Endocrinol Metab 2004;89:1089-95.

[27] Eggermont J, Donnez J, Casanas-Roux F, Scholtes H, Van Langendonckt A. Time course of pelvic endometriotic lesion revascularization in a nude mouse model. Fertil Steril 2005;84:492-9.

[28] Goffm F, Munaut C, Frankenne F, Perrier d'Hauterive S, Beliard A, Fridman V, et al. Expression pattern of metalloproteinases and tissue inhibitors of matrix-metalloproteinases in cycling human endometrium. Biol Reprod 2003;69:976-84.

[29] Chung HW, Lee JY, Moon HS, Hur SE, Park MH, Wen Y, et al. Matrix metalloproteinase-2, membranous type 1 matrix metalloproteinase, and tissue inhibitor of metalloproteinase-2 expression in ectopic and eutopic endometrium. Fertil Steril 2002;78:787-95.

[30] Gescher DM, Haensel A, Meyhofer-Malik A, Malik E. The importance of angiogenesis for the pathogenesis of endometriosis. Zentralbl Gynakol 2003;125:243-6.

[31] Red-Horse K, Rivera J, Schanz A, Zhou Y, Winn V, Kapidzic M, et al. Cytotrophoblast induction of arterial apoptosis and lymphangiongenesis in an in vivo model of human placentation. J Clin Invest 2006;116: 2643-52.

[32] Abrao MS, Podgaec S, Dias JA, Averbach M, Garry R, Ferraz Silva LF, et al. Deeply infiltrating endometriosis affecting the rectum and lymph nodes. Fertil Steril 2006;86:543-7. 